for the therapy of metabolic diseases but never delivered. In 2005, the "hormone" obestatin was reported as a previously overlooked by-product of preproghrelin cleavage with the ability to induce satiety in the CNS (12). Since then, scientists in the field have struggled to repeat the effects of obestatin on food intake or body weight seen in the previous report as well as to confirm its ability to bind and activate G protein-coupled receptor 39 (12).

With all of the GI hormones identified to date, it has turned out that their actions in the CNS are neither restricted to receptor activation in a single brain region nor limited to the modulation of a single physiological function. GLP-1 receptors, for example, are widely distributed throughout the brain and have been linked to visceral illness, anxiety, glucocorticoid secretion, and even promotion of neuronal survival (13, 14). As another example, ghrelin appears to act in key regions of the hippocampus, olfactory cortex, and ventral tegmental area to regulate memory, reward functions, and even mood (15-17), in addition to affecting energy balance in the hypothalamus. So while the data generated by Valentino et al. (5) point to a discrete satiety-inducing function for uroguanylin within the hypothalamus, it seems likely that it will act at multiple target sites and serve a wider range of yet to be determined physiological processes. Whether these will limit or enhance the therapeutic potential of this intriguing system awaits further study. However, with this important discovery by Valentino et al. (5), yet another potential tool has been provided to harness the biology of the gut-brain axis to help fill the medical need to treat obesity and diabetes.

\section{Acknowledgments}

Randy J. Seeley was supported by NIDDK DK54890. The authors would like to acknowledge J. Walker for facilitating discussions related to this commentary.

Address correspondence to: Randy J. Seeley, Metabolic Diseases Institute, 2170 E. Galbraith Rd., University of Cincinnati, Cincinnati, Ohio 45237, USA. Phone: 513.558.6665; Fax: 513.558.0187; E-mail: randy.seeley@uc.edu.

1. Wren AM. Gut and hormones and obesity. Front Horm Res. 2008;36:165-181.

2. Strader AD, Woods SC. Gastrointestinal hormones and food intake. Gastroenterology. 2005; 128(1):175-191.

3. Buchwald $\mathrm{H}$, et al. Weight and type 2 diabetes after bariatric surgery: systematic review and meta-analysis. Am J Med. 2009;122(3):248-256.

4. Thaler JP, Cummings DE. Minireview: Hormonal and metabolic mechanisms of diabetes remission after gastrointestinal surgery. Endocrinology. 2009;150(6):2518-2525.
5. Valentino MA, et al. A uroguanylin-GUCY2C endocrine axis regulates feeding in mice. $J$ Clin Invest. 2011;121(9):3578-3588.

6. Sindic A, Schlatter E. Renal electrolyte effects of guanylin and uroguanylin. Curr Opin Nephrol Hypertens. 2007;16(1):10-15.

7. Forte LR Jr. Uroguanylin and guanylin peptides: pharmacology and experimental therapeutics. Pharmacol Ther. 2004;104(2):137-162.

8. You YJ, Kim J, Raizen DM, Avery L. Insulin, cGMP, and TGF-beta signals regulate food intake and quiescence in C. elegans: a model for satiety. Cell Metab. 2008;7(3):249-257.

9. Drucker DJ. The biology of incretin hormones. Cell Metab. 2006;3(3):153-165

10. Renshaw D, Batterham RL. Peptide YY: a potential therapy for obesity. Curr Drug Targets. 2005; 6(2):171-179.

11. Kirchner $\mathrm{H}$, et al. GOAT links dietary lipids with the endocrine control of energy balance. Nat Med. 2009;15(7):741-745

12. Zhang JV, et al. Obestatin, a peptide encoded by the ghrelin gene, opposes ghrelin's effects on food intake. Science. 2005;310(5750):996-999.

13. Kinzig KP, et al. CNS glucagon-like peptide-1 receptors mediate endocrine and anxiety responses to interoceptive and psychogenic stressors. J Neurosci. 2003;23(15):6163-6170.

14. Kinzig KP, D'Alessio DA, Seeley RJ. The diverse roles of specific GLP-1 receptors in the control of food intake and the response to visceral illness. J Neurosci. 2002;22(23):10470-10476.

15. Chuang JC, Zigman JM. Ghrelin's roles in stress, mood, and anxiety regulation. Int J Pept. 2010; 2010:460549.

16. Lutter M, et al. The orexigenic hormone ghrelin defends against depressive symptoms of chronic stress. Nat Neurosci. 2008;11(7):752-753.

17. Tong J, et al. Ghrelin enhances olfactory sensitivity and exploratory sniffing in rodents and humans. J Neurosci. 2011;31(15):5841-5846

\title{
Behind an enteric neuron there may lie a glial cell
}

\author{
Michael D. Gershon
}

Department of Pathology and Cell Biology, Columbia University, College of Physicians and Surgeons, New York, New York, USA.

\begin{abstract}
The enteric nervous system (ENS) controls the gastrointestinal system. Enteric glia have long been regarded as the essential "glue" of the ENS. Now, however, two independent reports in this issue of the JCI provide compelling evidence that mouse enteric glia can also be neuronal precursors. These reports show that enteric glia give rise to neurons in vitro and that neurogenesis can be experimentally induced to occur in vivo in the adult mouse ENS. Unfortunately, glia do not constitutively replace neurons, and neurogenesis is not easily provoked. Although these new observations make it clear that clinical trials using glia to replace enteric neurons are more than premature, they are enticing for future research.
\end{abstract}

The enteric nervous system (ENS) is a neural crest-derived division of the autonomic nervous system that is essential for life (1).

Conflict of interest: The author has declared that no conflict of interest exists.

Citation for this article: J Clin Invest. 2011; 121(9):3386-3389. doi:10.1172/JCI59573.
It controls the gastrointestinal (GI) system and comprises a large number of neurons and glia that are organized into complex networks of interconnected ganglia distributed throughout the gut wall. Enteric neurons cluster into two plexi: the myenteric plexus, which is situated between the inner circular and outer longitudinal layers of the muscularis externa; and the submucosal plexus, which is located within the dense connective tissue between the muscularis externa and the mucosa.

One of the many functions of the GI system controlled by the ENS is motility, and thereby GI transit. As a result, aganglionosis of the bowel, whether congenital (as in Hirschsprung disease) or acquired (as in Chagas disease), leads to intestinal obstruction $(2,3)$. Nerve bundles, however, are present in the aganglionic segments of colon in individuals with Hirschsprung disease, meaning that nerves are not, by themselves, sufficient for GI transit (4). Nerve cell bodies and the complex microcircuits of the ENS, which uniquely enable it to control GI motility and secretion in the absence of CNS input, are required for normal GI tran- 
A Myenteric plexus (in situ)

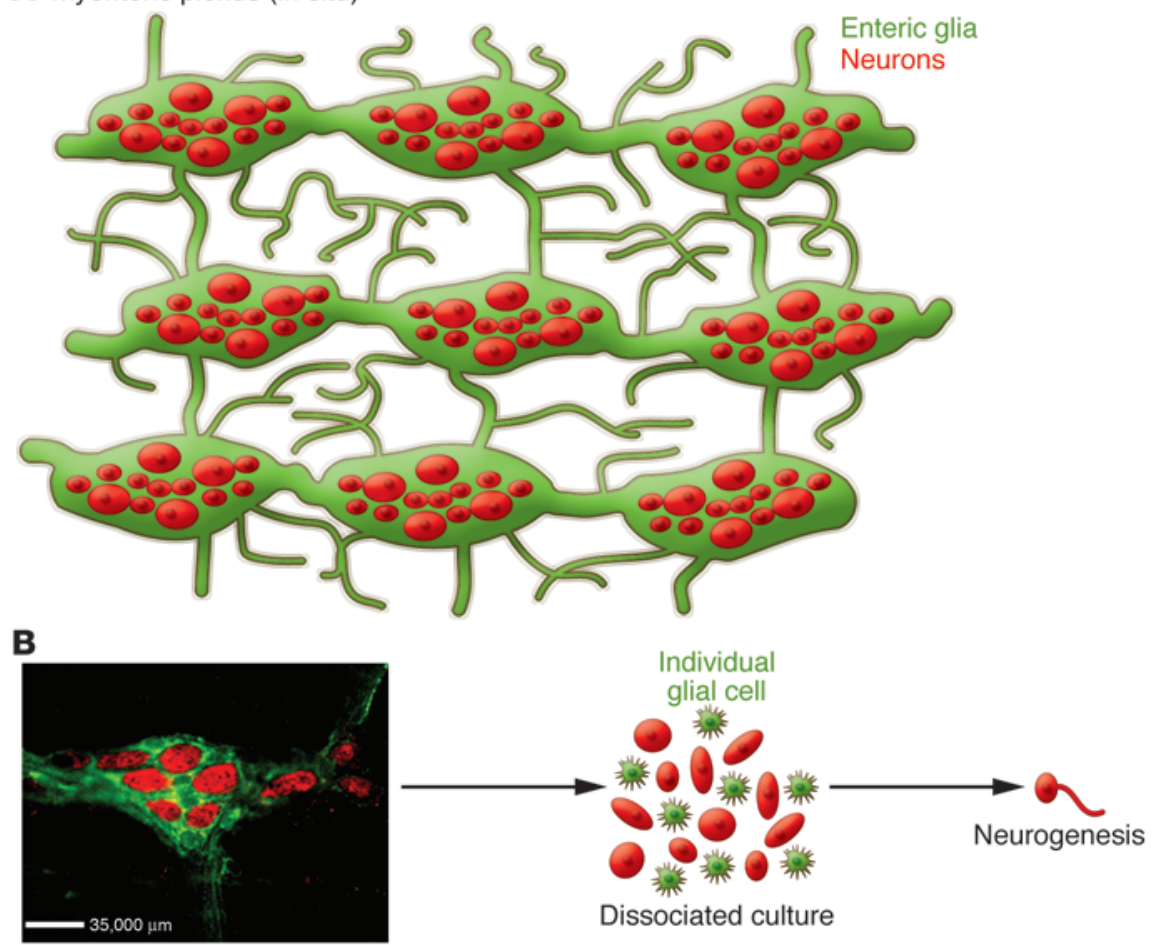

C

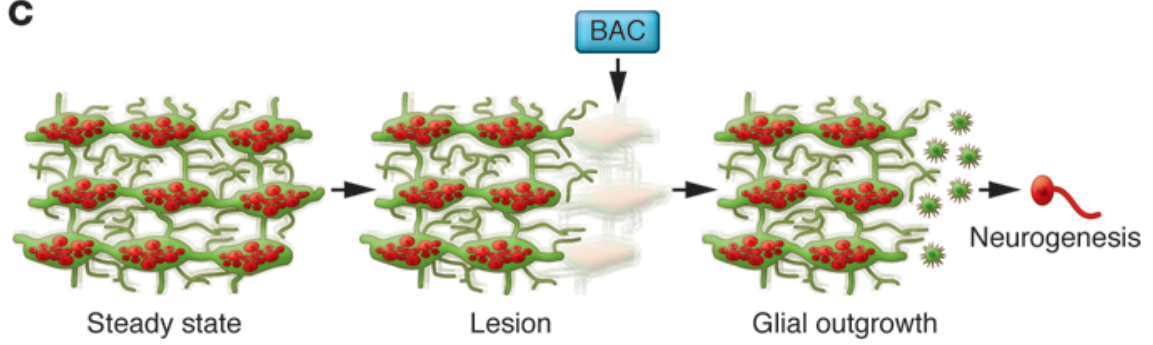

Figure 1

Disruption of contact between cells may initiate neurogenesis from precursors that express enteric glial markers. (A) A cartoon depicting the intact myenteric plexus as it appears in a whole mount of a laminar preparation of the intestinal wall. The neurons (red) are shown as they appear when a marker, such as $\mathrm{HuC} / \mathrm{D}$, that is restricted to the perikarya is immunocytochemically demonstrated. The neurons are surrounded by a dense network of enteric glia (green), which are depicted as they would appear when demonstrated immunocytochemically with antibodies specific for a marker such as $\mathrm{S} 100 \beta$. Neural pathways interconnect the ganglia, but they and the tertiary plexus of nerve fibers that innervate the longitudinal muscle are seen only with the glia marker because axons do not contain HuC/D. Boundaries between adjacent glial cells are not readily detected. At steady state, as shown, there is no neurogenesis. (B) An intact ganglion (left) is depicted in an actual photomicrograph immunostained to visualize HuC/D and S100 $\beta$ simultaneously (scale bar: $35 \mu \mathrm{m}$ ). When ganglia are dissociated (right) and cultured, the networks of glia and neurons are disrupted and a subset of glia give rise to neurons. $5-\mathrm{HT}$, which is present in culture media, may also activate $5-\mathrm{HT}_{4}$ receptors to stimulate neurogenesis. (C) Although neurogenesis from glia does not occur in the intact ENS at steady state (left), its disruption by BAC (center) leads to a glial outgrowth, and a subset of these glial cells, now no longer in contact with each other, may initiate neurogenesis.

sit (5). Enteric neurons, moreover, require support from the vast numbers of glia, which, in addition to "gluing" together the various components of the ENS, nurture, defend, and insulate the neurons, as well as clean up debris should neurons die. protect them from mechanical trauma; moreover, the gut is contractile, subjecting enteric neurons to levels of pressure that CNS neurons do not have to face. The enteric microbiome is also potentially dangerous to the ENS and is opposed by immune/inflammatory mechanisms that often operate with collateral damage to surrounding tissue and the ENS (6). Loss of enteric neurons is therefore a significant problem with which evolution has had to contend. Replacement of neurons that die is a potential solution that evolution might have used and which, if understood, could perhaps be therapeutically exploited.

\section{Adult neurogenesis: precedents}

Neurogenesis occurs in the adult CNS from stem cells that are located in the subgranular zone of the hippocampal dentate gyrus and in the subventricular zone adjacent to the lateral ventricles (7). Because neural crest-derived stem cells persist in the adult gut (8), one might therefore anticipate that, as in the CNS, neurogenesis occurs in adult bowel; however, pulse-chase studies in mice given thymidine analogs suggest that enteric neurons arise from mid-gestation through the first three weeks of postnatal life, but not thereafter $(9,10)$. Neurogenic stem cells, moreover, have been difficult to visualize in the adult gut because markers have been lacking. Recently, however, it has become clear that neurogenesis can occur in the adult ENS, although the circumstances that provoke it remain elusive.

\section{Adult neurogenesis: enteric glia as precursors of neurons}

In this issue of the JCI, Joseph et al. (11) and Laranjeira et al. (12) independently report compelling evidence that enteric glia are capable of giving rise to neurons in the bowel of adult mice, at least under certain restricted conditions. Joseph et al. prospectively used flow cytometry with antibodies specific for $\alpha 2$ integrin (also known as CD49b) to isolate cells that give rise to neurons in vitro from laminar preparations containing the myenteric plexus (ref. 11 and Figure 1A). The immunoselected neuronal precursors expressed the common neurotrophin receptor p75 (also known as p $^{\mathrm{NTR}}$ ), glial fibrillary acidic protein (GFAP), S100 $\beta$, Sox 10 , and nestin, which, in the adult gut, are identifiers of glia. Laranjeira et al. employed genetic fate mapping and found that most enteric neurons are derived from Sox10-expressing precursors that are present in the gut 
at mid-gestation, that neurogenesis then declines as a function of age, but that Sox10-expressing neural precursors, which express glial markers, persist (12). Both groups conclude that enteric glia are neurogenic, and both agree that glia give rise to neurons in vitro. GFAP, however, is also expressed by neurogenic progenitor cells in the CNS, raising the question of whether the cells that give rise to neurons in the adult gut are banal enteric glia or a subset of precursors that share markers with them (13). The new studies thus mean that a new and previously unsuspected function of neurogenesis must be added to the tasks - such as nurturing, insulation, cleaning, and defense of the ENS - that enteric glia undertake. Glia are thus more than just "glue" for the ENS, which lacks intraganglionic connective tissue.

\section{Adult neurogenesis: an in vivo phenomenon?}

Neurogenesis in the adult bowel may have gained a new player in enteric glia, but neither Joseph et al. nor Laranjeira et al. settle the role played by neurogenesis in homeostasis of the adult gut. In fact, it seems clear that constitutive neurogenesis does not occur. That conclusion was reached in an earlier investigation by Liu et al. (14) and is common to the work of both Joseph et al. and Laranjeira et al. Liu et al. discovered that activation of 5-hydroxytryptamine 4 $\left(5-\mathrm{HT}_{4}\right)$ receptors induces adult enteric neurogenesis in the mouse (14); moreover, although the ENS gained neurons through four months of age in wild-type mice, it did not do so in mice lacking $5-\mathrm{HT}_{4}$ receptors. Matsuyoshi et al., furthermore, found that production of new neurons, initiated in mice with a $5-\mathrm{HT}_{4}$ agonist, hastened the return of function after rectal transection and end-to-end anastomosis were used to disrupt ENS continuity (15).

Laranjeira et al. (12) report that ablation of existing enteric neurons with benzalkonium chloride (BAC), like $5-\mathrm{HT}_{4}$ agonism, provokes neurogenesis in the adult mouse gut. Joseph et al. (11), however, were able to obtain this result with BAC in only one animal, and they further report a remarkably long and diverse list of stimuli that failed to evoke neurogenesis in vivo. Joseph et al. thus conclude that although enteric glia have the potential to form both neurons and glia, in adult mice they are fated "to form mainly glia.” In contrast, Laranjeira et al. echo the $5-\mathrm{HT}_{4}$ data in concluding that the neurogenic potential of glia can be unlocked in vivo, in their case by "tissue dissociation or injury."

Taken together, available studies $(11,12$, $14,15)$ now make clear that neurogenesis can, in fact, occur in the adult mouse ENS, but that it does not happen spontaneously and it is not trivial to induce. 5-HT is endogenous to the mucosa and ENS and, through $5-\mathrm{HT}_{4}$ receptors, it is a potential initiator of neurogenesis $(14,15)$; nevertheless, it is not clear that the precursors for $5-\mathrm{HT}_{4}$-evoked neurogenesis are enteric glia, although the reports of Joseph et al. (11) and Laranjeira et al. (12) are certainly consistent with this possibility. Newly born neurons found after $5-\mathrm{HT}_{4}$ agonism first appear in extraganglionic locations and slowly migrate into the myenteric plexus (14). When first detected, these cells, like enteric glia, express Sox10; however, they are not glia but committed neurons: they lack glial markers (such as GFAP and S100 $\beta$ ) and they express neuronal markers (such as Phox $2 \mathrm{~b}, \mathrm{HuC} / \mathrm{D}$, and doublecortin). Their unidentified predecessors might have been glia, but this remains an open question that awaits the development of a marker for adult enteric neurogenic stem cells. CD49b sufficed to enable Joseph et al. (11) to immunoselect neurogenic stem cells, but CD49b is widely expressed and is not a stem cell marker. Laranjeira et al. (12) argue that the enteric glial population of the adult gut is unlikely to be homogeneous. They thus acknowledge that it is unclear whether all glial cells are neurogenic precursors or whether the neurogenic potential is restricted to a small fraction of these cells. They might, for example, be analogous to the GFAP-expressing neuronal precursors of the CNS (13). Extraganglionic cells that express glial markers are found throughout the gut, in part supporting nerve fibers (Figure 1); however, given the observations of Liu et al. (14), it is possible that the neurogenic precursors that express glial markers are preferentially extraganglionic.

\section{Adult neurogenesis: therapeutic potential in the gut}

It is exciting to wonder whether the new revelations about adult neurogenesis in the mouse embodied in the outstanding articles by Laranjeira et al. (12) and Joseph et al. (11) can be harnessed therapeutically. Patients with conditions such as Hirschsprung disease, chronic intestinal pseudoobstruction, diabetic neuropathy, gastroparesis, and even chronic constipation might benefit from a means to produce new neurons. Stem cell therapy is enticing, but so many problems confront the use of exogenous stem cells that learning how to activate neurogenic precursors in a patient's own bowel is an attractive alternative. Although the work of Laranjeira et al. (12) and Joseph et al. (11) suggests that potentially abundant endogenous precursors that have been hiding in plain sight exist in enteric glia, these articles also make it clear that turning enteric glia into enteric neurons in situ is not going to be easy. The subset of glia that is neurogenic may be small, and the many failed attempts that Joseph et al. made to induce neurons to develop in the adult mouse gut are discouraging. Laranjeira et al. suggest that disruption of contact, as occurs when glia are grown in dissociated cultures (Figure 1B) or after ENS ablation (Figure 1C), might provoke glia to give rise to neurons. Dissociation of glial networks in vivo, however, is a challenging therapeutic approach. On the other hand, the complex culture media used in the studies of Laranjeira et al. and Joseph et al. contain 5-HT, which, rather than dissociation, might have been responsible for the predilection detected in both studies for neurons to arise in vitro. It is worth noting that although the ENS appears normal in mice lacking tryptophan hydroxylase 1 (TPH1), which lack mucosal 5-HT, enteric neuronal numbers are reduced in TPH2-deficient animals, which lack neuronal 5-HT (16). Conceivably, ENS injury may provoke surviving serotonergic neurons to activate neurogenesis from 5- $\mathrm{HT}_{4}$-expressing glial progenitors. The studies of Matsuyoshi et al. (15) are compatible with speculation that stimulation of such a mechanism accelerates healing of an injured ENS. Whatever the role of 5-HT may be, Laranjeira et al. and Joseph et al. identify harnessing the endogenous neurogenic potential of enteric glia for therapeutic advantage as a worthy, if difficult, goal for neurogastroenterologists to achieve.

\section{Acknowledgments}

The author's work is funded by NIH grants NS12969 and NS15547.

Address correspondence to: Michael D. Gershon, Department of Pathology and Cell Biology, Columbia University, College of Physicians and Surgeons, 630 West 168th Street, New York, New York 10128, USA. Phone: 212.305.3447; Fax: 212.305.3973; E-mail:mdg4@columbia.edu. 1. Furness JB. The Enteric Nervous System. Malden, Mas-
sachusetts, USA: Blackwell Publishing; 2006.

2. Amiel J, et al. Hirschsprung disease, associated 
syndromes and genetics: a review. J Med Genet. 2008;45(1):1-14.

3. Matsuda NM, Miller SM, Evora PR. The chronic gastrointestinal manifestations of Chagas disease. Clinics (Sao Paulo). 2009;64(12):1219-1224.

4. Moore SW, Johnson G. Acetylcholinesterase in Hirschsprung's disease. Pediatr Surg Int. 2005; 21(4):255-263

5. Wood JD. Enteric nervous system: reflexes, pattern generators and motility. Curr Opin Gastroenterol. 2008;24(2):149-158.

6. Linden DR, et al. Indiscriminate loss of myenteric neurones in the TNBS-inflamed guinea-pig distal colon. Neurogastroenterol Motil. 2005;17(5):751-760.

7. Mu Y, Lee SW, Gage FH. Signaling in adult neurogenesis. Curr Opin Neurobiol. 2010;20(4):416-423.

8. Kruger GM, Mosher JT, Bixby S, Joseph N, Iwashita
T, Morrison SJ. Neural crest stem cells persist in the adult gut but undergo changes in self-renewal, neuronal subtype potential, and factor responsiveness. Neuron. 2002;35(4):657-669.

9. Pham TD, Gershon MD, Rothman TP. Time of origin of neurons in the murine enteric nervous system. J Comp Neurol. 1991;314(4):789-798.

10. Chalazonitis A, et al. Bone morphogenetic protein regulation of enteric neuronal phenotypic diversity: relationship to timing of cell cycle exit. J Comp Neurol. 2008;509(5):474-492.

11. Joseph NM, He S, Quintana E, Kim Y-G, Núñez G, Morrison SJ. Enteric glia are multipotent in culture but primarily form glia in the adult rodent gut. J Clin Invest. 2011;121(9):3398-3411.

12. Laranjeira C, et al. Glial cells in the mouse enteric nervous system can undergo neurogenesis in response to injury. J Clin Invest. 2011;121(9):3412-3424.

13. Garcia AD, Doan NB, Imura T, Bush TG, Sofroniew MV. GFAP-expressing progenitors are the principal source of constitutive neurogenesis in adult mouse forebrain. Nat Neurosci. 2004;7(11):1233-1241.

14. Liu MT, Kuan YH, Wang J, Hen R, Gershon MD. 5-HT4 receptor-mediated neuroprotection and neurogenesis in the enteric nervous system of adult mice. J Neurosci. 2009;29(31):9683-9699.

15. Matsuyoshi H, et al. A 5-HT(4)-receptor activationinduced neural plasticity enhances in vivo reconstructs of enteric nerve circuit insult. Neurogastroenterol Motil. 2010;22(7):806-813.

16. Li Z, et al. Essential roles of enteric neuronal serotonin in gastrointestinal motility and the development/survival of enteric dopaminergic neurons. J Neurosci. 2011;31(24):8998-9009.

\title{
Parkin reinvents itself to regulate fatty acid metabolism by tagging CD36
}

\author{
Nada A. Abumrad ${ }^{1}$ and Darren J. Moore ${ }^{2}$ \\ ${ }^{1}$ Washington University School of Medicine, Department of Medicine, Center for Human Nutrition, St. Louis, Missouri, USA. \\ 2Ecole Polytechnique Fédérale de Lausanne (EPFL), Brain Mind Institute, School of Life Sciences, Lausanne, Switzerland.
}

\begin{abstract}
Parkinson disease (PD) is a relatively common neurodegenerative disorder characterized by the progressive degeneration of dopaminergic neurons in the substantia nigra. About $5 \%-10 \%$ of PD cases are inherited. Mutations in the Parkin gene, which encodes a protein that can function as an E3 ubiquitin ligase, are a common cause of familial PD. Such mutations act in a lossof-function manner and impair the ability of the encoded protein to mediate substrate ubiquitination, although the subsequent molecular pathway that precipitates neuronal degeneration is poorly defined. In this issue of the JCI, Kim and colleagues describe painstaking evidence using a number of dissecting approaches in intact animals and cultured cells to functionally link Parkin and the class B scavenger receptor CD36, suggesting a novel and complex connection between PD and fatty acid metabolism.
\end{abstract}

Parkinson disease (PD) affects $1 \%-2 \%$ of the world's population over the age of 65 years. It is more common in developed countries where people live longer. PD is characterized by the progressive degeneration of dopaminergic neurons in the substantia nigra, which leads to diminished striatal dopamine levels and the appearance of intracytoplasmic proteinaceous inclusions, termed Lewy bodies, in surviving brainstem neurons. The cardinal motor symptoms of PD include bradykinesia, resting tremor, muscular rigidity, and postural instability. There are also nonmotor symptoms, which include autonomic, cognitive, and psychiatric disturbances. About

Conflict of interest: The authors have declared that no conflict of interest exists.

Citation for this article: J Clin Invest. 2011; 121(9):3389-3392. doi:10.1172/JCI59219.
$5 \%-10 \%$ of PD cases are inherited, with at least 16 chromosomal loci (PARK1-16) associated with these familial forms of disease (1). Mutations in the $\alpha$-synuclein gene (SNCA, which falls into both the PARK1 and PARK4 loci) and the leucine-rich repeat kinase 2 gene (LRRK2, which accounts for the PARK8 locus) unambiguously cause autosomal dominant PD, whereas mutations in the Parkin gene (which accounts for the PARK2 locus), the PTEN-induced putative kinase 1 gene (PINK1, which accounts for the PARK6 locus), the DJ-1 gene (which accounts for the PARK7 locus), and the ATPase type 13A2 gene (ATP13A2, which accounts for the PARK9 locus) cause autosomal recessive forms of the disease (1). Idiopathic PD is considered to result from a combination of genetic susceptibility, aging, and environmental factors (for example, pesticides) (2).

\section{Parkin: a ubiquitin ligase linked to PD}

Mutations in the Parkin gene on chromosome 6 cause autosomal recessive, juvenile-onset parkinsonism (AR-JP), the most common form of early-onset disease $(3,4)$. The Parkin gene encodes a 465 amino acid protein consisting of a ubiquitin-like domain and a RING-box domain containing two RING finger motifs separated by an in-between-RING finger motif (Figure 1 and ref. 5). Similar to other RING-box proteins, Parkin can function as an E3 ubiquitin ligase to catalyze, in cooperation with E1-activating and E2-conjugating enzymes, the covalent attachment of the carboxyl terminal glycine residue of ubiquitin to an internal lysine residue of protein substrates (5). Parkin can link substrates to chains of ubiquitin polymerized through different lysine residues of the ubiquitin components, with K48-linked chains targeting substrates for proteasomal degradation and K63-linked chains acting as a nondegradative signal (5). Parkin can also mediate monoubiquitination of proteins and itself (6). Familial mutations in Parkin act in a loss-of-function manner and impair substrate ubiquitination, although the subsequent molecular pathway that precipitates neuronal degeneration is poorly defined. In this issue of the JCI, Kim et al. have identified a new substrate of Parkin-mediated ubiquitination that provides a novel and 